In Table 2 values for the power emitted by the three different radiation mechanisms in a laser produced plasma are given for three different temperatures. We assumed a radiating volume $V$ of $10^{-7} \mathrm{~cm}^{3}$ which is a representative value for the volume of the heating zone. The electron densities were taken from the expression given in Eq. (8). $P V$ is the total power of radiation emitted by the plasma. For comparison the laser power $P_{\mathrm{L}}$ which is necessary to heat the plasma up to the indicated temperature is given.

We see from this comparison that in general radiation losses may be neglected. Only at low electron temperatures and with targets with elements of high $Z$ losses due to the line radiation are considerable.

${ }^{27}$ G. Elwert, Z. Naturforsch. 7 a, 432 [1952].

\title{
Temperature and Expansion Energy of Laser Produced Plasmas
}

\author{
II. Experiments \\ H. Puell, H. J. Neusser, and W. Kaiser \\ Physik-Department der Technischen Universität München \\ (Z. Naturforsch. 25 a, 1815-1822 [1970] ; received 25 September 1970)
}

\begin{abstract}
Experiments on laser produced plasmas with light intensities between $2 \cdot 10^{11}$ and $5 \cdot 10^{12} \mathrm{~W} / \mathrm{cm}^{2}$ are reported. Measurements of the electron temperature and the expansion energy were performed. In the case of $\mathrm{LiD}$ targets the highest temperature observed was $200 \mathrm{eV}$. Using carbon targets a maximum temperature of $330 \mathrm{eV}$ was measured. The corresponding expansion energies reach values as high as $13 \mathrm{keV}$. The experimental results are in good agreement with a stationary, hydrodynamic theory. We conclude from our data that in our LiD and $\mathrm{C}$ plasmas ions and electrons have the same temperature.
\end{abstract}

The production of plasmas by irradiating plane, solid targets with intense light pulses has been investigated experimentally by a number of authors (l. c. $\left.{ }^{1-7}\right)$. The main interest of these studies is to determine plasma parameters such as electron density and temperature, ion expansion energy, and total number of particles produced during the interaction. We wish to report results obtained by irradiating plane, solid targets of lithium deuteride ${ }^{8}$ and carbon with a powerful giant pulse ruby laser. The experimentally determined values for electron temperature and ion expansion energy will be compared with the results derived from the theory presented in a previous paper ${ }^{9}$ (later referred to as A) . In Section I we shall summarize the most important results derived in A. Our experimental set up and the results are presented in Sections II and III respectively. In the Appendix, a detailed discussion is presented on the X-ray method which was used for determining the electron temperature.

${ }^{1}$ For reference of earlier publications see H. OPOWER, W. Kaiser, H. Puell, and W. Heinicke, Z. Naturforsch. 22 a, 1392 [1967].

2 N. G. Basov, V. A. Boiko, V. A. Dementev, O. N. KroKHIN, and G. V. SKLIzKov, Sov. Phys. JETP 24, 659 [1967].

3 E. W. Sucov, J. L. PACK, A. V. Phelps, and A. G. ENGelHARDT, Phys. Fluids 10, 2035 [1967].

4 J. L. Bobin, F. Floux, P. Langer, and H. Pingerol, Phys. Letters 28 A, 398 [1968].

\section{Theoretical Remarks}

In A) we developed a hydrodynamic model describing the plasma production as a steady state process. Taking into account the finite dimension of the focal spot we were able to divide the plasma in three regions: a) the undisturbed target, b) a zone of one-dimensional plasma flow extending from the target surface out to a distance $R$ ( $R$ ist the focal spot radius), and c) a zone of three-dimensional plasma flow (see Fig. 1 in A). It was shown that during the simultaneous heating and expansion of the plasma the flow velocity $u$ reaches the local velocity of sound at the point $x=R$. Considering an energy balance between the incoming light flux and the thermal- and kinetic energy transported by the plasma flow, we were able to determine the highest plasma temperature $k T_{1}$, the total number of charges $N$ produced during the interaction, and ion expansion energy $E$ as a function of the incident light

5 B. C. Boland, F. E. Irons, and R. W. P. McWhirter, J. Phys. B 1, 1180 [1968].

6 C. Colin, Y. Durand, F. Floux, D. Guyot, P. Langer, and P. Veyrie, J. Appl. Phys. 39, 2991 [1968].

7 R. Sigel, Z. Naurforsch. 25 a, 488 [1970].

8 The LiD single crystals were kindly provided by Prof. Dr. S. HaussüHL.

${ }^{9}$ H. Puell, Z. Naturforsch. 25 a, 1807 [1970] ; preceding publication. 
flux $\Phi_{0}$. The results are summarized in the following equations:

$$
\begin{aligned}
& k T_{1}=\alpha^{-2 / 3}(3 M C R / 50)^{2 / 9} \Phi_{0}^{4 / 9}, \\
& N \quad=\pi R^{2} \alpha^{-1 / 3}(2 / 3 M C R)^{2 / 9} \int_{-\infty}^{\infty}\left(\Phi_{0} / 5\right)^{5 / 9} \mathrm{~d} t, \\
& E \quad=5 Z \alpha k T_{1}=5 Z \alpha^{1 / 3}(3 M C R / 50)^{2 / 9} \Phi_{0}{ }^{4 / 9} ;
\end{aligned}
$$

$M$ and $Z$ are the mass and the charge of the ions. In the case of a plasma with different ion species one has to take the average values for these quantities as pointed out in A). The constant $C$ results from the absorption by inverse bremsstrahlung ${ }^{10}$. Its numerical value in CGS units is

$$
C=2.5 \cdot 10^{-55}\left(\omega_{\mathrm{R}} / \omega\right)^{2}
$$

where $\omega_{\mathrm{R}}$ is the frequency of the ruby laser $\left(\omega_{\mathrm{R}}=\right.$ $2.7 \cdot 10^{15} \mathrm{sec}^{-1}$ ) and $\omega$ is the frequency of light under consideration. $\alpha$ is a factor which takes into account whether the ions are thermalized with the electrons: $\alpha=(Z+1) / Z$ for $T_{\mathrm{i}}=T_{\mathrm{e}}$ and $\alpha=1$ for $T_{\mathrm{i}}=0$.

It was pointed out in A) that Eqs. (1), (2), and (3) are only valid for light fluxes $\Phi_{0}$ less than a critical light flux $\Phi_{0}{ }^{\prime}$, where

$$
\Phi_{0}{ }^{\prime}=25 C R n_{\mathrm{ep}}^{3}\left(5 \alpha^{3} Z^{3} / 12 M\right)^{1 / 2} .
$$

$n_{\mathrm{ep}}$ is the electron density at which the corresponding plasma frequency equals the frequency of the incident light. Its value for the frequencies of the two commonly used laser is $n_{\text {ep }}$ (ruby) $=2.3 \cdot 10^{21}$ $\mathrm{cm}^{-3}$ and $n_{\mathrm{ep}}(\mathrm{Nd}$ :glass $)=10^{21} \mathrm{~cm}^{-3}$. Our experiments are performed with light intensities below the limit given by $\Phi_{0}{ }^{\prime}$; e. g. for a fully ionized carbon plasma a value of $8 \cdot 10^{13} \mathrm{~W} / \mathrm{cm}^{2}$ is estimated from Eq. (4).

\section{Experimental}

The experimental system for measuring electron temperatures and ion expansion energies is depicted schematically in Fig. 1. The laser consisted of an oscillator and a four stage amplifier system. Two totally reflecting prisms formed the resonator of the oscillator. A small fraction of the light flux was deflected from the resonator by a glass plate. The active medium was a ruby crystal, $16 \mathrm{~cm}$ long and $0.8 \mathrm{~cm}$ in diameter. A cryptocyanine solution in methanol was used for Q. switching. The advantage of this type of oscillator is the fast risetime of the emitted light pulse ${ }^{11}$. In our

10 J. Dawson and C. Oberman, Phys. Fluids 5, 517 [1962]. 11 K. Darée and H. Puell, Z. Naturforsch. 25 a, 909 [1970].

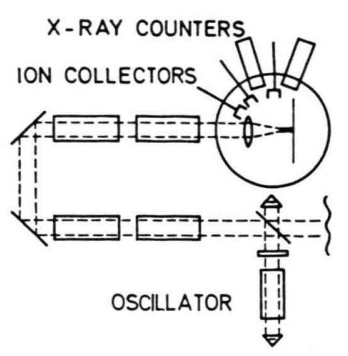

Fig. 1. Experimental system for the plasma production on plane solid targets.

experiments the oscillator emitted an exponentially rising light pulse with a risetime of 1.6 nsec. The output pulse had a peak power of $60 \mathrm{MW}$ and a halfwidth of 7 nsec. This signal was amplified by the amplifier system to a power level of approximately $1 \mathrm{GW}$.

Target, lens system, and measuring devices were placed in a chamber with a vacuum better than $10^{-4}$ $\mathrm{mm} \mathrm{Hg}$. The light beam was focused by a lens system with an aperture of 0.98 and a focal length of $7 \mathrm{~mm}$. The resulting focal spot diameter was estimated from the divergence of our laser beam (20 minutes of arc) to be $80 \mu$. The targets consisted of small rods of lithium deuteride or of carbon with a diameter of $100 \mu$. The expanding plasma produced at these targets was analyzed by time of flight measurements for determining the ion expansion energy and by X-ray measurements for determining the electron temperature in the plasma.

Electrostatic detectors were used for measuring the expansion velocity of the ions. An electric field of $150 \mathrm{~V} / \mathrm{cm}$ was arranged perpendicular to the plasma stream in order to extract the electrons from the beam and to measure a pure ion signal ${ }^{12}$. Three detectors were placed under angles of $7^{\circ}, 43^{\circ}$, and $90^{\circ}$ to the target normal in the same plane and in distances of $111 \mathrm{~mm}, 85 \mathrm{~mm}$, and $240 \mathrm{~mm}$ from the target. Severa! diaphragms limited their acceptance angle to about $10^{-4}$ steradians. The overall time constant of these collectors was less than 15 nsec. This time is short compared to the expected time of flight of the ions of several 100 nsec. The collectors present a time resolved signal of the incoming ions (the pulse duration of the laser is short compared to the times involved). From the measured time interval between the laser pulse and the arrival of the ions at the detector we calculate the energy of the ions, if their mass is known.

In Fig. 2 a typical signal of one of the ion collectors $\left(7^{\circ}\right)$ is shown. The small signal at the beginning of the oscilloscope trace indicates the time when the laser pulse hits the target. It is formed by the production of photoelectrons at the collector. The real ion signal shows two characteristic times: a) the beginning of the signal corresponds to the arival of the most energetic ions $\left(E_{\max }\right)$ at the detector and b) the maxi-

12 H. Opower and W. Press, Z. Naturforsch. 21 a, 344 [1966]. 


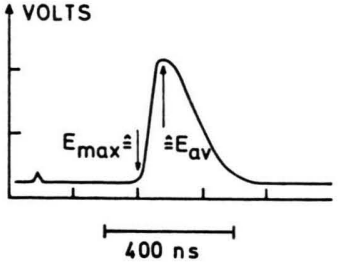

Fig. 2. Typical ion signal obtained from the charge collector in the $7^{\circ}$ direction. The voltage is proportional to the charge flux of the incoming ions.

mum of the signal is generated by the most abundant ions having an average energy $E_{\mathrm{av}}$. The height of the signal is proportional to the incoming charge flux $\mathrm{d} N / \mathrm{d} t$. The total number of charges emitted by the plasma into the acceptance angle of the detector is obtained by integrating over the whole signal.

The electron temperature was determined following a method first suggested by JAHODA et al. ${ }^{13}$. The relative transmission of two different foils to the $\mathrm{X}$-rays (bremsstrahlung) is measured. Using proper absorber materials the relative transmission is a strong function of the plasma temperature (as will be shown in detail in the appendix). In our experiments we used Al- and Be-foils of different thickness (Al: $15 \mu, 25 \mu$, and $50 \mu$; $\mathrm{Be}: 80 \mu, 100 \mu, 300 \mu$, and $500 \mu)$. The transmitted X-rays were registered by two plastic scintillators and RCA $6342 \mathrm{~A}$ photomultipliers. The plastic scintillators were both coated with an Al layer $3000 \AA$ in thickness in order to eliminate effects due to stray light of the laser. The counters were placed at a distance of $110 \mathrm{~mm}$ from the target in a plane perpendicular to the target normal. Their solid angle was limited by diaphragms to $10^{-4}$ steradians. Because of the short pulse duration of the laser and the small plasma volume only integrated measurements in time and space were possible. It will be shown in the appendix that this system measures the maximum temperature of the plasma (except small local temperature fluctuations).

For a quantitative comparison of the experimental data with the theoretical results an accurate knowledge of the light intensity at the target is necessary. In our experiments we measured the power of the laser beam before entering the focusing system with a calibrated photodiode and displayed the signal on a fast oscilloscope. The risetime of the system was less than .4 nsec. In Fig. 3 a typical oscilloscope trace of a laser pulse is shown. The time scale is $10 \mathrm{nsec}$ per $\mathrm{cm}$. In each measurement, the energy of the light pulse was determined by a calibrated calorimeter.

To determine the diameter of the focal spot we measured the divergence $\varepsilon$ of our laser beam. By focusing the beam on a photographic plate with a lens of focal length $f=100 \mathrm{~cm}$ we determined the half angle of divergence to 20 minutes of arc. Using the relation $R=\varepsilon f$ we calculated the radius $R$ of the focal spot within the focal plane of our lens system $(f=7 \mathrm{~mm})$ to $40 \mu$.

13 F. C. Jahoda, E. M. Little, W. E. Quinn, G. A. Sawyer, and T. F. Stratton, Phys. Rev. 119, 843 [1960].

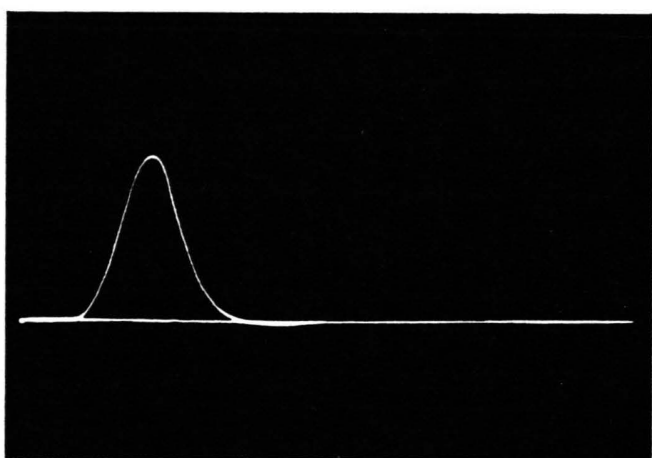

Fig. 3. Oscilloscope trace of a light pulse emitted by the laser system. Peak power $450 \mathrm{MW}$, time scale $10 \mathrm{nsec} / \mathrm{cm}$.

This value is in agreement with computer calculations of the focal spot diameter taking into account the spherical aberration and the coma of the lenssystem. The light intensity at the target was calculated from the measured input power and the focal spot size taking into account the losses of the optical system.

\section{Experimental Results}

\section{A) Electron Temperature}

Temperature measurements were performed over a wide range of light intensities on lithium deuteride and carbon plasmas. In the Appendix it is shown that our device measures the highest temperature in the plasma. We have to relate the measured temperatures to the light flux at the maximum of the laser pulse. In Fig. 4 and Fig. 5 our experimental results are plotted as a function of the incident light intensity. The highest temperature observed was $330 \mathrm{eV}$ in the case of carbon targets and $200 \mathrm{eV}$ in the case of lithium deuteride targets. The experimental points in Fig. 4 (C) represents values averaged over a

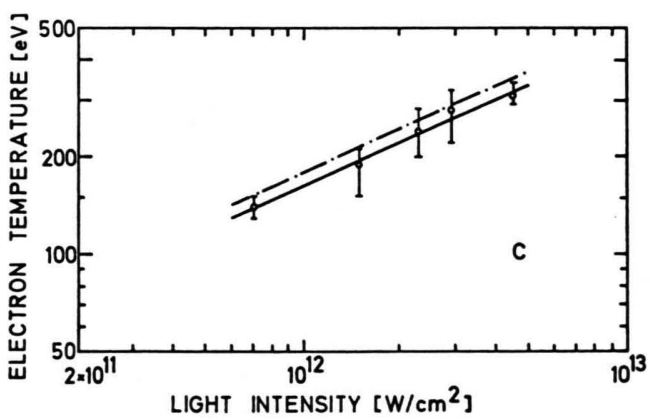

Fig. 4. Measured electron temperatures versus incident laser intensities (carbon-target). Theoretical curves according to Eq. (1) are given for $T_{\mathrm{i}}=T_{\mathrm{e}}(\longrightarrow)$ and for $T_{\mathrm{i}}=0(-\cdot-\cdot-)$. 


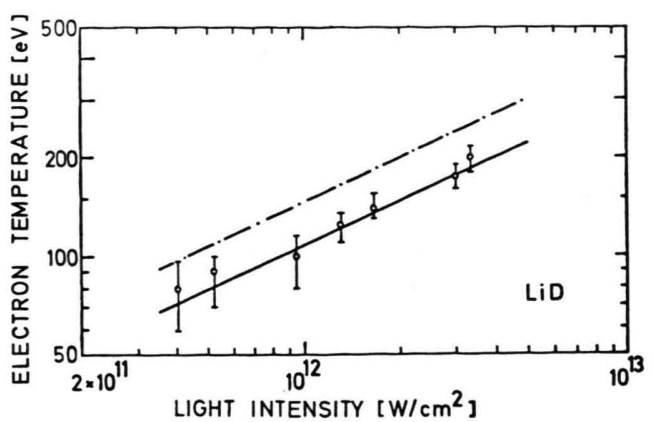

Fig. 5. Measured electron temperatures versus incident laser intensities (LiD-target). Theoretical curves according to Eq. (1) are given for $T_{\mathrm{i}}=T_{\mathrm{e}}(\longrightarrow)$ and for $T_{\mathrm{i}}=0(-\cdot-\cdot-)$.

number of shots. The error bars indicate the maximum deviation from the average value observed in the experiment. In the case of LiD targets (Fig. 5) the experimental points represent the results of single shots. Here the error bars indicate the accuracy of the measurements mainly determined by fluctuations of the multiplier gain.

In Fig. 4 and Fig. 5 the theoretical curves for the electron temperature are shown, calculated according to Eq. (1). They were obtained by inserting into Eq. (1) the experimental parameters: $R=40 \mu$, $Z=6$ in the case of carbon and $Z=2$ in the case of lithium deuteride. The solid curve is calculated for equal temperature of electrons and ions $\left(T_{\mathrm{i}}=T_{\mathrm{e}}\right)$, while the dash-dot curve is obtained assuming a negligible ion temperature $\left(T_{\mathrm{i}} \cong 0\right)$. The agreement of measured and calculated temperatures is very good. The results suggest that electrons and ions are thermalized in the plasma. This result was expected from an estimate of the energy equipartition time between electrons and ions in the plasma (see A).

The following point is of interest here. We have estimated the electron temperature of a carbonhydrogen plasma which was investigated by BOLAND et al. ${ }^{5}$. Inserting their experimental parameters $\Phi_{0}=3 \cdot 10^{11} \mathrm{~W} / \mathrm{cm}^{2}, R=150 \mu$, into Eq. (1) and considering the average mass $[M=(12+2) / 3]$ and average charge $[Z=(6+2) / 3]$ of a $\mathrm{C}-\mathrm{H}_{2}$ plasma we calculated an electron temperature of $95 \mathrm{eV}$. This value is in complete agreement with the $95 \pm 10 \mathrm{eV}$ determined from their spectroscopic measurements.

\section{B) Particle Number and Ion Expansion Energy}

From the time of flight measurements performed with the charge collectors we obtain two significant informations about the plasma: the total charge and the ion expansion energy in the plasma. As mentioned above the total charge of the ions emitted in the corresponding solid angle of the detector is estimated by integrating over the ion signal. In Fig. 6 a polar diagram of the number of charges per steradian for a carbon plasma is shown. The angular distribution was obtained from the signals of charge collectors placed at angles of $7^{\circ}, 43^{\circ}$, and $90^{\circ}$ to the target normal.

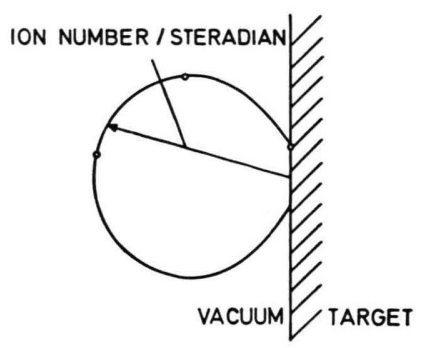

Fig. 6. Polar diagram of the measured total number of charges per steradian expanding from a carbon target at an incident laser intensity of $\Phi_{0}=2.5 \cdot 10^{12} \mathrm{~W} / \mathrm{cm}^{2}$.

We notice a preferential expansion of the plasma in the direction of the target normal similar to earlier findings ${ }^{12}$. If we assume a rotational symmetry of the expanding plasma around the target normal we are able to evaluate the total number of charges contained in the plasma. The resulting numbers of charges are presented in Fig. 7 as a function of the

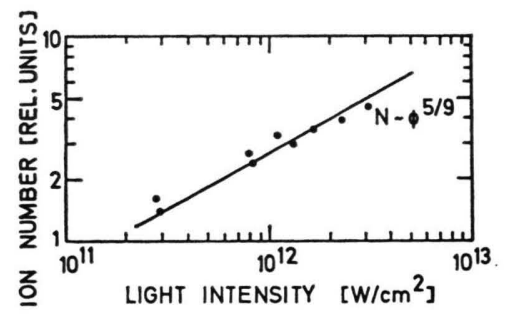

Fig. 7. Measured total number of charges expanding from a carbon target versus the incident laser intensity.

— Theoretical curve according to Eq. (2).

light intensity. Slight changes in pulse duration of the light pulses from shot to shot were taken into account by normalizing the experimental results with respect to the laser pulse halfwidth. The solid line represents the theoretically expected dependence resulting from Eq. (2). The agreement with the experiment is good. Relative values for the number of charges are given; for a quantitative treatment one has to study the problem of recombination in the plasma which is beyond the scope of this paper. 
The time dependence of the ion signal gives information of the expansion energy of the ions. We are mainly interested in the energy of the fastest ions $\left(E_{\max }\right)$ and in the energy of the most abundant ions $\left(E_{\text {av }}\right)$. In Fig. 8 a polar diagram for $E_{\max }$ is given. Similar to the case of the number of charges

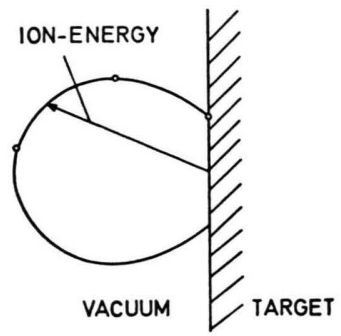

Fig. 8. Polar diagram of the measured ion expansion energies (carbon target, $\Phi_{0}=2.5 \cdot 10^{12} \mathrm{~W} / \mathrm{cm}^{2}$ ).

(Fig. 6) we notice a pronounced anisotropy. The most energetic ions are measured in the direction of the target normal. This observation results from the planary geometry of the target. It was pointed out by DAwSON et al. ${ }^{14}$ that the highest acceleration of the ions takes place in the direction of the smallest dimension within the plasma. In our theoretical model given in A) the preferential expansion of the plasma in the direction of the target normal was implicitly introduced by assuming a one-dimensional geometry at the beginning of the expansion. We anticipate that the theoretical values [Eq. (3)] are best compared with the ion energies measured in the forward direction $\left(7^{\circ}\right.$ detector $)$.

In Fig. 9 and Fig. 10 the measured ion expansion energies for carbon and lithium deuteride plasmas are plotted as a function of the incident light intensity. The solid line represents the theoretical values for the expansion energy calculated from Eq. (3) with $R=40 \mu, Z=6$ (C) and $Z=2$ (LiD) respectively. The dashed lines are just auxiliary lines showing that the values of $E_{\max }$ as well as $E_{\text {av }}$ follow the same power law. As expected, the theoretical curve lies somewhat below the experimental curve for $E_{\max }$. One has to bear in mind that only a small number of ions have the high energy $E_{\max }$.

The good agreement between theory and experiment is believed to confirm the model discussed in A). In particular the substantial difference between electron temperature and ion expansion energy

14 J. Dawson, P. Kaw, and B. Green, Phys. Fluids 12, 875 [1969].

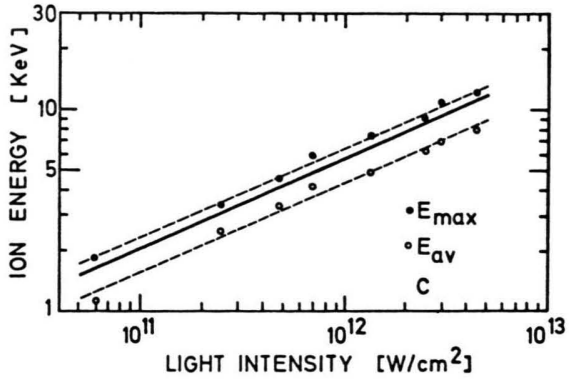

Fig. 9. Measured ion expansion energies versus incident laser intensities (carbon target). - Theoretical curve according to Eq. (3).

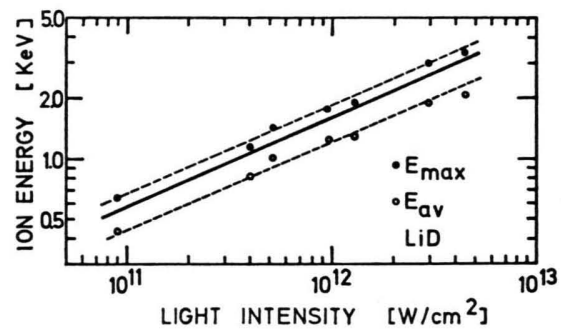

Fig. 10. Measured ion expansion energies versus incident laser intensities (LiD-target). - Theoretical curve ac cording to Eq. (3).

found experimentally is well accounted for by theory.

It should be pointed out that the assumptions made in the calculation concerning the charge of the ions $[Z=6$ (carbon) and $Z=2(\mathrm{LiD})]$ are justified in the case of the most energetic ions. An estimate of the degree of ionization (see appendix in A) indicates that for temperatures $>100 \mathrm{eV}$ a $\mathrm{LiD}$ plasma may be regarded as fully ionized. The same holds for a carbon plasma at temperatures $>200$ $\mathrm{eV}$. Even at temperatures of about $100 \mathrm{eV}\left(\Phi_{0} \cong\right.$ $3 \cdot 10^{11} \mathrm{~W} / \mathrm{cm}^{2}$ ) $8 \%$ of the carbon ions will be fully ionized. This estimate is in agreement with spectroscopic studies on laser produced $\mathrm{C}-\mathrm{H}_{2}$ plasmas ${ }^{5}$.

\section{Conclusions}

The experimental results represented here showed a satisfactory agreement with the theoretical predictions given in A. It is interesting that in the case of lithium deuteride agreement is achieved if the average mass and charge is used in the calculation. This fact has been suggested by theory. Experiments should be done with light intensities near the critical light flux $\Phi_{0}^{\prime}$ [see Eq. (4) ]. In this region one expects from theory the power law for the electron 
temperature and the ion expansion energy to change to $\Phi_{0}{ }^{2 / 3}$ (see discussion in A). Bobin et al. ${ }^{15} \mathrm{ob}$ served such a power law for temperature measurements in deuterium plasmas produced with a $\mathrm{Nd}$ : glass laser. Their experimental parameters indicated that their light intensity was near or above the critical value of $\Phi_{0}^{\prime}$.

\section{Acknowledgement}

The authors wish to thank H. SchülLER for the computer calculations of the transmission of foils to bremsstrahlung.

\section{Appendix \\ A) Foil Transmission Method for Temperature Measurements}

In this appendix we wish to discuss some details of our temperature measuring device, especially the influence of recombination radiation and line radiation and of the temporal and spatial integration of our system.

The foil transmission method ${ }^{13}$ employed in our experiments for determining the electron temperature is based on two facts:

a) The power of bremsstrahlung per unit wave length interval $P_{\mathrm{ff}}(\lambda)$ emitted by a plasma changes in the short wave length region exponentially with the plasma temperature $k T^{16}$

$$
P_{\mathrm{ff}}(\lambda) \propto \exp \{-h c / \lambda k T\} / \lambda^{2}(k T)^{1 / 2} .
$$

(For $\lambda \ll h c / k T$ the exponential factor dominates in the equation given above.)

b) The transmission of all materials increases in the short wave length region with decreasing wave length of the incident radiation (except at the different $\mathrm{K}, \mathrm{L}$, and $\mathrm{M}$ edges).

Because of these two facts foils from e. g. beryllium, carbon, aluminium or nickel act like monochromators for the incident bremsstrahlung. This can be readily seen by folding the bremsstrahlung spectrum with the absorption spectrum of the foil material. We will designate the center wave-length of the transmitted wavelength interval with $\lambda_{0}$. A small change in plasma temperature causes a shift in $\lambda_{0}$ and there will be, therefore, a considerable change in the transmitted intensity.

To determine the plasma temperature one measures the transmission of two different foils for bremsstrahlung. The plasma temperature is infered from the ratio of the transmitted signals, if the transmission of the two foils is known as a function of temperature. JAHODA et al. ${ }^{13}$ tabulated transmissions for $\mathrm{CH}_{2}, \mathrm{Be}$,

15 J. L. Bobin, F. Delobeau, G. DeGiovanni, C. FauquigNON, and F. Floux, Nucl. Fusion 9, 115 [1969].

16 G. Elwert, Z. Naturforsch. 9 a, 637 [1954].
$\mathrm{Al}$, and $\mathrm{Ni}$ foils of different thickness. More detailed data for the same materials are given by ELTON and ANDERSON ${ }^{17}$. To increase the accuracy of our temperature measurements we calculated the transmission $\mathcal{J}$ of any combination of different foils (on a computer). The transmission is calculated from the integral

$$
\mathcal{J}=\int_{0}^{\infty}\left\{\exp \left\{(-h v / k T)-\left[\sum_{(i)} \alpha_{i}(v) x_{i}\right]\right\} h \mathrm{~d} v\right\}(k T)^{-1} \text {. }
$$

The first term in the integrand is due to the brems. strahlung spectrum, whereas the second term takes into account the absorption of the foil materials. The normalizing factor $(k T)^{-1}$ results from the integration of the pure bremsstrahlung over the whole frequency range. $x_{i}$ is the thickness and $\alpha_{i}(v)$ the absorption coefficient of the $i$-th absorber material. Values for $\alpha_{i}(v)$ were taken from the curves compiled by GILMORE ${ }^{18}$.

Our numerical results on the transmission of various foils are in good agreement with the results given by Refs. ${ }^{13}$ and ${ }^{18}$. In our calculations a $3000 \AA$ thick layer of $\mathrm{Al}$, evaporated on the plastic scintillators for discrimination of stray light, was taken into account.

In Fig. 11 and Fig. 12 the calculated transmissions for various foils of beryllium and aluminium are shown as a function of plasma temperature. Aluminium foils

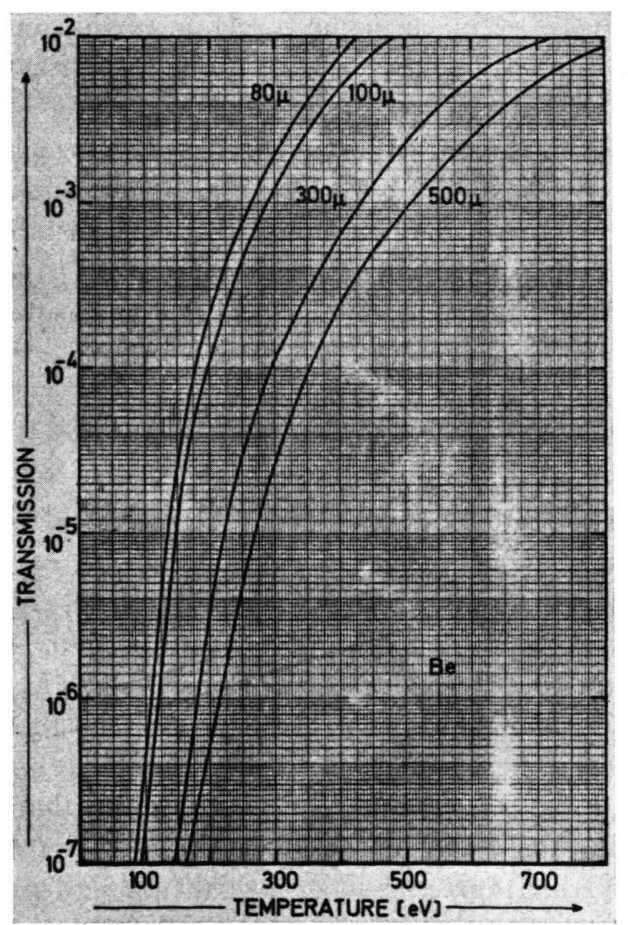

Fig. 11. Calculated transmission of Be-foils to bremsstrahlung versus plasma temperature.

17 R. C. Elton and A. D. Anderson, NRL Report 6541.

18 F. R. Gilmore, AECU Report 4353. 


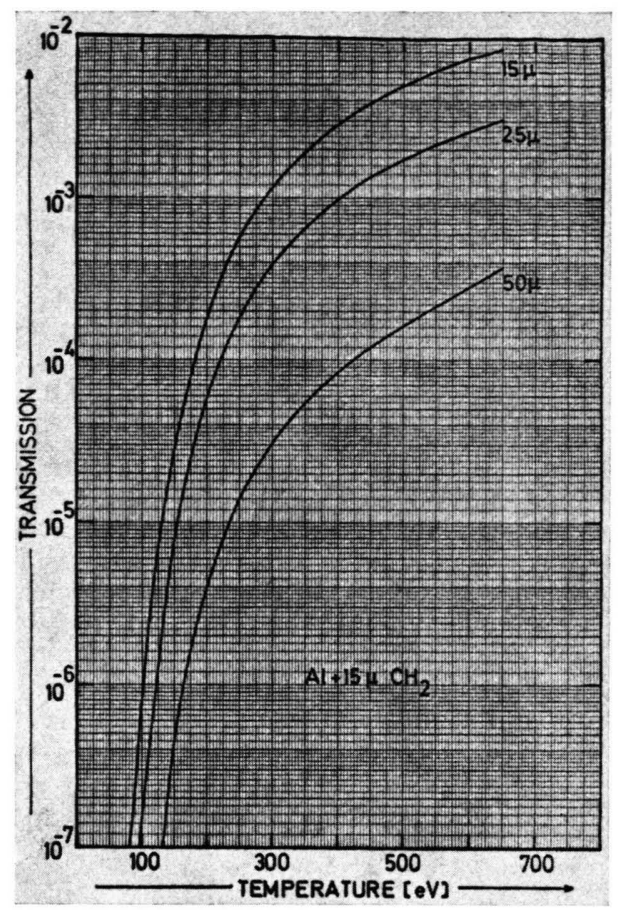

Fig. 12. Calculated transmission of $\mathrm{Al}+15 \mu \mathrm{CH}_{2}$ foils to bremsstrahlung versus plasma temperature.

were used in conjunction with polyethylene foils of $15 \mu$ thickness in order to reduce the enhanced transmission of the Al foils near their L edge when measuring low temperatures.

In Fig. 13 the relative transmission for different foil combinations are shown as a function of plasma temperature. Notice the pronounced change of the relative transmission with temperature. The accuracy of such temperature measurements might be as good, as $10 \%$ to $20 \%$. Other factors which affect the accuracy will be discussed in the next section.

In Tab. 1 we give foil combinations and temperature ranges used in our experiments. Severe difficulties arise for temperatures below $70 \mathrm{eV}$ on account of the line radiation of the plasma (see Sect. B).

It should be pointed out that the relative sensitivity of the registration system has to be determined for this type of measurements. In our experiments the multipliers were calibrated by measuring the transmission to bremsstrahlung through two identical foils (each counter was covered with $\left.15 \mu \mathrm{CH}_{2}+25 \mu \mathrm{Al}\right)$. The resulting relative sensitivity was $R_{1} / R_{2}=2.1 \pm .2$.

\begin{tabular}{cc}
\hline Foil combinations & Temperature range $(\mathrm{eV})$ \\
\hline $80 \mu \mathrm{Be} /\left(15 \mu \mathrm{Al}+15 \mu \mathrm{CH}_{2}\right)$ & $70-150$ \\
$100 \mu \mathrm{Be} /\left(15 \mu \mathrm{Al}+15 \mu \mathrm{CH}_{2}\right)$ & $100-200$ \\
$300 \mu \mathrm{Be} /\left(25 \mu \mathrm{Al}+15 \mu \mathrm{CH}_{2}\right)$ & $150-300$ \\
$500 \mu \mathrm{Be} /\left(50 \mu \mathrm{Al}+15 \mu \mathrm{CH}_{2}\right)$ & $270-500$ \\
\hline
\end{tabular}

Table 1. Temperature range in which various foil combinations were preferentially used for temperature measurements.

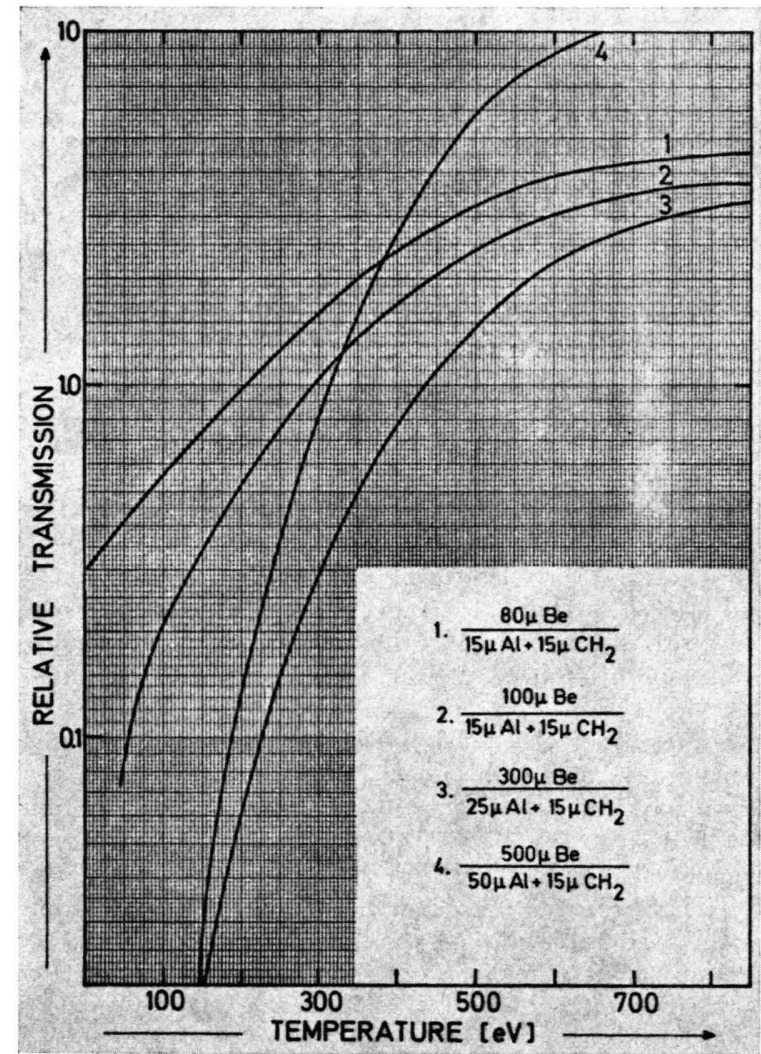

Fig. 13. Calculated relative transmissions of different foil combinations versus plasma temperature.

\section{B) Influence of Recombination and Line Radiation}

One of the fundamental approximations used in the foil transmission method is the assumption of a pure bremsstrahlung spectrum emitted by the plasma. But there is recombination and line radiation of substantial intensity emitted in certain cases (see appendix in A). We wish to show under which conditions this radiation can be neglected.

Recombination radiation and bremsstrahlung show the same spectral behaviour in the frequency region $h v>\chi_{\bar{n}}{ }^{17}$, where $\chi_{\bar{n}}$ is the ionization energy from the $\bar{n}$-th shell of an atom. In the case of $h v=\chi_{\bar{n}}$ there is a discontinuity in the recombination spectrum and no radiation is observed for $h v<\chi_{\bar{n}}$. Calculating the transmission of a foil for recombination radiation, analogous to Eq. (5), we notice that there will be no difference in the results compared to the bremsstrahlung, as long as the transmitted wave-length $\lambda_{0}$ is well below the wave length corresponding to the discontinuity of the recombination spectrum: $\lambda_{0} \ll h c / \chi_{\bar{n}}$. Let us consider an example: the transmission of a $15 \mu$ thick $\mathrm{Al}$ foil. At a plasma temperature of $100 \mathrm{eV}$ the transmitted bremsstrahlung has a wave length $\lambda_{0} \cong 10 \AA$. This value is well below the edge of the recombination spectrum from a carbon plasma ( $\left.h c / \chi_{\bar{n}} \cong 25 \AA\right)$. Using 
thicker foils and higher temperature the inequality will become even more favorable. In short, we may neglect the influence of recombination radiation in the foil transmission method, if the foils used are thick enough and the temperature to be observed is sufficiently high.

Now the influence of line radiation should be considered. This type of radiation may be quite intense if the plasma contains ions of high atomic number and if the plasma temperature is low. In this case the intensity may become higher than the corresponding bremsstrahlung intensity.

A carbon plasma of a temperature of $100 \mathrm{eV}$ emits, for instance, about $5 \cdot 10^{13} \mathrm{~W} / \mathrm{cm}^{3}$ as line radiation. The radiation is located mainly at a wave length of $40 \AA$ (resonance line of $\mathrm{C}^{4+}$ ); approximately $20 \%$ of the power is emitted at $33 \AA$ (resonance line of $\mathrm{C}^{5+}$ ). The absorption coefficient of aluminium and polyethylene foils at this wave-length is $\alpha_{\mathrm{Al}}(33 \AA)=1.9 \cdot 10^{4} \mathrm{~cm}^{-1}$ and $\alpha_{\mathrm{CH}_{2}}(33 \AA)=2.3 \cdot 10^{4} \mathrm{~cm}^{-1}$ respectively ${ }^{19}$. From these values one estimates the transmitted power of line radiation through a $15 \mu \mathrm{Al}+15 \mu \mathrm{CH}_{2}$ foil combination to be $3 \cdot 10^{-14} \mathrm{~W} / \mathrm{cm}^{3}$.

On contrast the total power radiated by the same plasma as bremsstrahlung is $2 \cdot 10^{11} \mathrm{~W} / \mathrm{cm}^{3}$. The transmission of the above mentioned foils to this radiation is $7.5 \cdot 10^{-7}$ (see Fig. 12); the transmitted power of bremsstrahlung is $1.5 \cdot 10^{5} \mathrm{~W} / \mathrm{cm}^{3}$.

The reason for the nearly complete discrimination of the line radiation by the foils under consideration one has to seek in the rapid increase of the absorption coefficient with increasing wavelength. In the case of bremsstrahlung mainly the short wavelength portion of the spectrum centered around $\lambda_{0}$ is transmitted through the foils (in our example one finds $\lambda_{0} \cong 9 \AA$ ).

Therefore, we conclude that in order to make the line radiation negligible the foil thickness has to be chosen in such a way that the most effectively transmitted wavelength $\lambda_{0}$ of the bremsstrahlung is less than the wavelength of the line radiation. In the case of a carbon plasma this condition is fulfilled for temperatures $>100 \mathrm{eV}$ and $\mathrm{Al}$-foils $>10 \mu$.

\section{C) Time and Space Integrating Measurements}

In our experiments time and space integrated measurements were performed because of the short laser pulse duration $\tau$ and the small plasma volume. First, we consider the influence of the time integration on our results. Let us assume a gaussian time dependence of the light intensity $\Phi_{0}$ and correspondingly of the electron temperature. According to Eq. (1) we write: $k T=A \Phi_{0}{ }^{4 / 9}(t)=A \Phi_{0}{ }^{4 / 8} \exp \left\{-(t / \tau)^{2} 4 / 9\right\}$

$$
A=(3 M C R / 50)^{2 / 9} \alpha^{-2 / 3} \text {. }
$$

The time integrated transmission $\mathcal{J}^{\prime}$ of a foil is obtained by inserting the time dependent expression for the temperature into Eq. (5) and integrating over the time

$$
\mathcal{J}^{\prime}=\frac{\int_{0}^{\infty} \int_{-\infty}^{\infty} \exp \{[-h v / k T(t)]-\alpha(v) x\} h \mathrm{~d} v \mathrm{~d} t}{\int_{-\infty}^{\infty} k T(t) \mathrm{d} t} .
$$

Using our numerical results of Eq. (5) we find:

$$
\mathcal{J}^{\prime}=\frac{\int_{-\infty}^{\infty} k T \mathcal{T}(k T) \mathrm{d} t}{\int_{-\infty}^{\infty} k T \mathrm{~d} t} .
$$

Here $\mathcal{T}(k T)$ is the transmission of the foil shown in Fig. 11 and Fig. 12. From these curves an empirical relation for the transmission as a function of temperature is determined.

$$
\mathcal{J}(k T) \propto \exp \left\{a(k T)^{-3 / 4}\right\} .
$$

Inserting Eq. (9) and Eq. (6) into Eq. (8) and integrating over the time we get approximately

$$
\mathcal{T}^{\prime} \cong \frac{\exp \left\{-a A^{-3 / 4} \Phi_{0}^{-1 / 3}\right\}}{\left(1+\frac{3}{4} a A^{-3 / 4} \Phi_{0}-1 / 3\right)^{1 / 2}} .
$$

Bearing in mind that $A \Phi_{0}{ }^{4 / 9}$ is equal to the temperature $k T_{1}$ at the time $t=0$ [see Eq. (6)] and that $k T_{1}$ is the highest temperature in the plasma we rewrite Eq. (10) using Eq. (9) :

$$
\mathcal{T}^{\prime}=\mathcal{J}\left(k T_{1}\right) / \sqrt{1-\frac{3}{4} \ln \left[\mathcal{J}\left(k T_{1}\right)\right]} .
$$

The relative transmission of two different foils $a$ and $b$ for a time integrating measuring device is:

$$
\frac{\mathcal{J}_{\mathrm{a}^{\prime}}}{\mathcal{J}_{\mathrm{b}^{\prime}}}=\frac{\mathcal{T}_{\mathrm{a}}\left(k T_{1}\right)}{\mathcal{J}_{\mathrm{b}}\left(k T_{1}\right)} \sqrt{\frac{1-\frac{3}{4} \ln \left[\mathcal{T}_{\mathrm{b}}\left(k T_{1}\right)\right]}{1-\frac{3}{4} \ln \left[\mathcal{T}_{\mathrm{a}}\left(k T_{1}\right)\right]}} .
$$

Using Eq. (12) one may evaluate the highest temperature $k T_{1}$ of the plasma with a time integrating registration system. In most cases one can use $\mathcal{J}_{\mathrm{a}}^{\prime} / \mathcal{T}_{\mathrm{b}}{ }^{\prime}$ directly as a measure for $k T_{1}$, since the square root factor is near unity if the transmission of both foils is not very different.

To estimate the influence of the space integration on our results one proceeds in a similar way than above. One has to take into account the spatial density distribution since the power of bremsstrahlung is proportional to the density squared. Assuming reasonable density gradients within the plasma one can show that the highest plasma temperature is measured as long as the transmission of the two foils is not very different. 